\title{
Effect of Long-Range Interactions on the Multicritical Behavior of Homogeneous Systems
}

\author{
S.V. Belin* \\ Omsk State University, 55-a, pr. Mira, Omsk, Russia, 644077 \\
}

(Dated: November 19, 2018)

\begin{abstract}
A field-theoretic approach is applied to describe behavior of homogeneous three-dimensional systems with long-range interactions defined by two order parameters at bicritical and tetracritical points. Renormalization- group equations are analyzed in the two-loop approximation by using the PadeBorel summation technique. The fixed points corresponding to various types of multicritical behavior are determined. It is shown that effects due to long-range interactions can be responsible for a change from bicritical to tetracritical behavior.
\end{abstract}

PACS numbers: 64.60.-i

It was shown in [1] that effects due to a long-range interaction described by the power law $r^{D \sigma}$ with $\sigma<2$ are responsible for a change in critical behavior. It was also revealed that the system is described by mean-field critical exponents.

This paper deals with effects of long-range interaction on systems described by two fluctuating order parameters. The phase diagrams of such systems can contain bicritical and tetracritical points. In the former case, a multicritical point is the point of intersection of two second-order phase transition curves and one first order phase transition curve. In the latter case, it is the point of intersection of four second-order phase transition curves. In the neighborhood of a multicritical point, the system exhibits critical behavior characterized by competing types of ordering. Whereas one critical parameter is replaced by the other at a bicritical point, the ordering types can coexist in a mixed phase at a tetracritical point. Such systems can be described in terms of two order parameters that are transformed under different irreducible representations.

The model Hamiltonian is written as

$$
\begin{aligned}
& H_{0}=\frac{1}{2} \int d^{D} q\left(\tau_{1}+q^{\sigma}\right) \Phi_{q} \Phi_{-q} \\
& +\frac{1}{2} \int d^{D} q\left(\tau_{2}+q^{a}\right) \Psi_{q} \Psi_{-q} \\
& +u_{01} \int d^{D}\left\{q_{i}\right\}\left(\Phi_{q 1} \Phi_{q 2}\right)\left(\Phi_{q 3} \Phi_{-q 1-q 2-q 3}\right) \\
& +u_{02} \int d^{D}\left\{q_{i}\right\}\left(\Psi_{q 1} \Psi_{q 2}\right)\left(\Psi_{q 3} \Psi_{-q 1-q 2-q 3}\right) \\
& +2 u_{03} \int d^{D}\left\{q_{i}\right\}\left(\Phi_{q 1} \Phi_{q 2}\right)\left(\Psi_{q 3} \Psi_{-q 1-q 2-q 3}\right)
\end{aligned}
$$

where $\Phi$ and $\Psi$ are fluctuating order parameters, $u_{01}$ and $u_{02}$ are positive constants, $\tau_{1} \sim\left|T-T_{c 1}\right| / T_{c 1}$, $\tau_{2} \sim\left|T-T_{c 2}\right| / T_{c 2}, T_{c 1}$ and $T_{c 2}$ are the corresponding phase-transition temperatures, and $\sigma$ is the long-range parameter.

*Electronic address: belim@univer.omsk.su
This Hamiltonian admits a wide diversity of multicritical points. The conditions for tetracritical and bicritical behavior are $u_{3}^{2}<u_{1} u_{2}$ and $u_{3}^{2} \geq u_{1} u_{2}$, respectively.

In the field-theoretic approach [3], the asymptotic critical behavior and structure of phase diagrams in the fluctuation region are governed by the CallanSymanczyk equation for the vertex parts of irreducible Green functions. The $\beta$ and $\gamma$ functions contained in the CallanSymanczyk equation for renormalized interaction vertexes $u_{1}, u_{2}, u_{3}$ are calculated by a standard method based on the Feynman diagram technique and a renormalization procedure [4]. As a result, the following expressions for $\beta$ functions are obtained in the two-loop approximation:

$$
\begin{aligned}
& \beta_{u 1}=(2 \sigma-D)\left(-u_{1}+36 J_{0} u_{1}^{2}+4 J_{0} u_{3}^{2}\right. \\
& -1728\left(2 J_{1}-J_{0}^{2}-\frac{2}{9} G\right) u_{1}^{3} \\
& -192\left(2 J_{1}-J_{0}^{2}-\frac{2}{9} G\right) u_{1} u_{3}^{2} \\
& \left.-64\left(2 J_{1}-J_{0}^{2}\right) u_{3}^{3}\right) \\
& \beta_{u 2}=(2 \sigma-D)\left(-u_{2}+36 J_{0} u_{2}^{2}+4 J_{0} u_{3}^{2}\right. \\
& -1728\left(2 J_{1}-J_{0}^{2}-\frac{2}{9} G\right) u_{2}^{3} \\
& -192\left(2 J_{1}-J_{0}^{2}-\frac{2}{9} G\right) u_{2} u_{3}^{2} \\
& \left.-64\left(2 J_{1}-J_{0}^{2}\right) u_{3}^{3}\right), \\
& \beta_{u 3}=(2 \sigma-D)\left(-u_{3}+16 J_{0} u_{3}^{2}+12 J_{0} u_{1} u_{3}+12 J_{0} u_{2} u_{3}\right. \\
& -320\left(2 J_{1}-J_{0}^{2}-\frac{2}{5} G\right) u_{3}^{3}- \\
& -288\left(2 J_{1}-J_{0}^{2}-\frac{2}{3} G\right) u_{1}^{2} u_{3} \\
& -288\left(2 J_{1}-J_{0}^{2}-\frac{2}{3} G\right) u_{2}^{2} u_{3} \\
& -576\left(2 J_{1}-J_{0}^{2}\right) u_{1} u_{3}^{2}- \\
& \left.-576\left(2 J_{1}-J_{0}^{2}\right) u_{2} u_{3}^{2}\right) \\
& -
\end{aligned}
$$




$$
\begin{aligned}
J_{1}= & \int \frac{d^{D} q d^{D} p}{\left(1+|\vec{q}|^{\sigma}\right)^{2}\left(1+|\vec{p}|^{\sigma}\right)\left(1+\left|q^{2}+p^{2}+2 \vec{p} \vec{q}\right|^{\sigma / 2}\right)}, \\
J_{0}= & \int \frac{d^{D} q}{\left(1+|\vec{q}|^{\sigma}\right)^{2}}, \\
G= & -\frac{\partial}{\partial|\vec{k}|^{\sigma}} \int \frac{d^{D} q d^{D} p}{\left(1+\left|q^{2}+k^{2}+2 \vec{k} \vec{q}\right|^{\sigma}\right)\left(1+|\vec{p}|^{\sigma}\right)} \\
& \cdot \frac{1}{\left(1+\left|q^{2}+p^{2}+2 \vec{p} \vec{q}\right|^{\sigma / 2}\right)}
\end{aligned}
$$

In terms of the new effective interaction vertices

$$
v_{1}=u_{1} \cdot J_{0}, \quad v_{2}=u_{2} \cdot J_{0}, \quad v_{3}=u_{3} \cdot J_{0}
$$

the $\beta$ functions are expressed as

$$
\begin{aligned}
& \beta_{1}=(2 \sigma-D)\left(-v_{1}+36 v_{1}^{2}+4 v_{3}^{2}\right. \\
& -1728\left(2 \widetilde{J_{1}}-1-\frac{2}{9} \widetilde{G}\right) u_{1}^{3} \\
& -192\left(2 \widetilde{J_{1}}-1-\frac{2}{9} \widetilde{G}\right) v_{1} v_{3}^{2} \\
& \left.-64\left(2 \widetilde{J_{1}}-1\right) v_{3}^{3}\right), \\
& \beta_{2}=(2 \sigma-D)\left(-v_{2}+36 v_{2}^{2}+4 v_{3}^{2}\right. \\
& -1728\left(2 \widetilde{J_{1}}-1-\frac{2}{9} \widetilde{G}\right) v_{2}^{3} \\
& -192\left(2 \widetilde{J_{1}}-1-\frac{2}{9} \widetilde{G}\right) v_{2} v_{3}^{2} \\
& \left.-64\left(2 \widetilde{J_{1}}-1\right) v_{3}^{3}\right), \\
& \beta_{u 3}=(2 \sigma-D)\left(-v_{3}+16 v_{3}^{2}+12 v_{1} v_{3}+12 v_{2} v_{3}\right. \\
& -320\left(2 \widetilde{J_{1}}-1-\frac{2}{5} \widetilde{G}\right) v_{3}^{3}- \\
& -288\left(2 \widetilde{J_{1}}-1-\frac{2}{3} \widetilde{G}\right) v_{1}^{2} v_{3} \\
& -288\left(2 \widetilde{J_{1}}-1-\frac{2}{3} \widetilde{G}\right) v_{2}^{2} v_{3}-576\left(2 \widetilde{J_{1}}-1\right) v_{1} v_{3}^{2} \\
& \left.-576\left(2 \widetilde{J_{1}}-1\right) v_{2} v_{3}^{2}\right), \\
& \widetilde{J_{1}}=\widetilde{J_{1} / J_{0}^{2}} \widetilde{G}=G / J_{0}^{2} .
\end{aligned}
$$

This redefinition is meaningful for $\sigma \leq D / 2$. In this case, $J_{0}, J_{1}$ and $G$ are divergent functions. Introducing the cutoff parameter $\Lambda$, we obtain finite expressions for the ratios $J_{1} / J_{0}^{2}$ and $G / J_{0}^{2}$ as $\Lambda \rightarrow \infty$.

The integrals are performed numerically. For $\sigma \leq D / 2$, a sequence of $J_{1} / J_{0}^{2}$ and $G / J_{0}^{2}$ corresponding to various values of $\Lambda$ is calculated and extrapolated to infinity.

It is well known that perturbation-theory series are asymptotic and expressions (4) cannot be applied directly since the interaction vertexes for order-parameter fluctuations in the fluctuation region are too large. For this reason, the required physical information was extracted from these expressions by applying the PadeBorel method extended to the four-parameter case. The appropriate di-

\begin{tabular}{|c|c|c|c|c|c|c|}
\hline$\sigma$ & $v_{1}^{*}$ & $v_{2}^{*}$ & $v_{3}^{*}$ & $b_{1}$ & $b_{2}$ & $b_{3}$ \\
\hline 1.9 & 0.035842 & 0.035842 & 0.039202 & 0.069 & 0.505 & 0.702 \\
1.8 & 0.033682 & 0.033682 & 0.034575 & 0.090 & 0.571 & 0.753 \\
1.7 & 0.031287 & 0.031287 & 0.031334 & 0.113 & 0.629 & 0.809 \\
1.6 & 0.027427 & 0.027427 & 0.026699 & 0.157 & 0.738 & 0.919 \\
1.5 & 0.026514 & 0.026514 & 0.025973 & 0.171 & 0.762 & 0.949 \\
\hline
\end{tabular}

rect and inverse Borel transforms have the form

$$
\begin{aligned}
& f(v 1, v 2, v 3)=\sum_{i_{1}, i_{2}, i_{3}} c_{i_{1} i_{2} i_{3}} v_{1}^{i_{1}} v_{2}^{i_{2}} v_{3}^{i_{3}} \\
& =\int_{0}^{\infty} e^{-t} F\left(v_{1} t, v_{2} t, v_{3} t, z_{1} t, z_{2} t, w_{1} t, w_{2} t\right) d t, \\
& F(v 1, v 2, v 3)=\sum_{i_{1}, i_{2}, i_{3}} \frac{c_{i_{1}, i_{2}, i_{3}}}{\left(i_{1}+i_{2}+i_{3}\right) !} v_{1}^{i_{1}} v_{2}^{i_{2}} v_{3}^{i_{3}} .
\end{aligned}
$$

To obtain an analytic continuation of the Borel transform of a function, a series in an auxiliary variable is introduced:

$$
\tilde{F}\left(v_{1}, v_{2}, v_{3}, \theta\right)=\sum_{k=0}^{\infty} \theta^{k} \sum_{i_{1}, i_{2}, i_{3}} \frac{c_{i_{1} i_{2} i_{3}}}{k !} v_{1}^{i_{1}} v_{2}^{i_{2}} v_{3}^{i_{3}} \delta_{i_{1}+i_{2}+i_{3}, k},
$$

The Pade approximant $[\mathrm{L} / \mathrm{M}]$ is applied to this series at $\theta=1$. The $[2 / 1]$ approximant is used to calculate the $\beta$ functions in the two-loop approximation. The critical behavior is completely determined by the stable fixed point $\left(v_{1}^{*}, v_{2}^{*}, v_{3}^{*}\right)$ satisfying the system of equations

$$
\beta_{i}\left(v_{1}^{*}, v_{2}^{*}, v_{3}^{*}\right)=0 \quad(i=1,2,3) .
$$

The requirement of stability of a fixed point reduces to the condition that the eigenvalues $b_{i}$ of the matrix

$$
B_{i, j}=\frac{\partial \beta_{i}\left(v_{1}^{*}, v_{2}^{*}, v_{3}^{*}\right)}{\partial v_{j}} \quad(i=1,2,3)
$$

lie in the right half-plane.

The resulting system of summed $\beta$ functions has a wide diversity of fixed points lying in the physical region of vertexes with $v_{i} \geq 0$. A complete analysis of the fixed points corresponding to the behavior of only one order parameter was presented in [1]. Here, the simultaneous critical behavior of both order parameters is considered. The stable fixed points and eigenvalues of the stability matrix are listed in the table.

An analysis of the critical points and their stability leads to certain conclusions. When $\sigma>1.6$, bicritical behavior is observed $v_{3}^{2} \geq v_{1} v_{2}$ ), as in the case of a shortrange interaction. When $1.5<a \leq 1.6$, tetracritical behavior is observed $\left(v_{3}^{2}<v_{1} v_{2}\right)$.

Thus, effects due to long-range interaction are responsible for a change from bicritical to tetracritical behavior when $1.5<a \leq 1.6$.

The work is supported by Russian Foundation for Basic Research N 04-02-16002. 
[1] S. V. Belim, Pisma Zh. ksp. Teor. Fiz. 77, 118 (2003) [JETP Lett. 77, 112 (2003)].

[2] V. V. Prudnikov, P. V. Prudnikov, and A. A. Fedorenko, Fiz. Tverd. Tela (St. Petersburg) 42, 158 (2000) [Phys. Solid State 42, 165 (2000)].

[3] D. Amit, Field Theory the Renormalization Group and Critical Phenomena (McGraw-Hill, New York, 1976).

[4] J. Zinn-Justin, Quantum Field Theory and Critical Phenomena (Clarendon Press, Oxford, 1989). 